\title{
Validity and reliability of Tamil version of strengths and difficulties questionnaire self-report
}

\author{
Pathumanabhan Lukumar ${ }^{1}$, Kumudu Wijewardana ${ }^{2}$, Johanna Hermansson ${ }^{3}$ and Gunilla Lindmark ${ }^{4}$
}

(Index words: SDQ, validity, reliability, mental health problems, adolescent, Tamil)

\begin{abstract}
Background Child and adolescent mental health services are scarce in Sri Lanka. Moreover there are no validated instruments to measure the mental health problems of Tamil speaking adolescents in Sri Lanka. Hence, strengths and difficulties questionnaire (SDQ) self-report was translated into Tamil and validated in the District of Jaffna, Sri Lanka.

Methods SDQ self-report was translated from English to Tamil using standard translation and back translation method. The Tamil version of the SDQ self-report was validated against a gold standard (ie. diagnosis made by a consultant psychiatrist). Both clinic and community samples were included in the validation study. Thirty-five adolescents (11-16 years) attending a psychiatric clinic and 91 adolescents from the community were included. The consultant psychiatrist made the diagnosis independent of the SDQ responses. Receiver operating characteristics (ROC) curve was used to measure the validity of SDQ. Reliability was measured using internal consistency.
\end{abstract}

Results The ROC curve indicated high validity for the identification of adolescents with mental health problems: area under the curve $0.87(95 \%-\mathrm{Cl}=0.79-0.93)$. International cut-off point of 15 gave a sensitivity of $69.4 \%$ and specificity of $92.2 \%$. Cronbach's alpha values (0.67-0.78) were good in all subscales and total difficulties scale.

Conclusion The SDQ self-report Tamil version can be used effectively for screening of adolescents to identify mental health problems as well as for research purposes.

\section{Introduction}

Child and adolescent mental health services are scarce in Sri Lanka. There are no validated instruments to measure mental health problems of Tamil-speaking adolescents in Sri Lanka. Therefore, it is necessary to translate and validate an instrument to assess mental health problems of Tamil-speaking adolescents.

The two most frequently used instruments for measuring psychopathological symptoms in children and

${ }^{1}$ Department of Community Medicine, Facutly of Medicine, University of Jaffna, Sri Lanka. ${ }^{2}$ Dean, Faculty of Graduate Studies, University of Sri Jayewardenepura, Gangodawila, Nugegoda, Sri Lanka. ${ }^{3}$ Child and Adolescent Psychiatrist, Stockholm, Sweden. ${ }^{4}$ International Maternal and Child Health, University of Uppsala, Sweden.

Correspondence: PL, e-mail <lukumar@hotmail.com>. Received 27 September and accepted 29 September 2007. Competing interests: none declared. 
adolescents are the Rutter and Achenbach questionnaires. The Rutter questionnaires are brief rating scales to be completed by parents and teachers that have proven to be reliable and valid indices of child psychopathology in many contexts [1]. Since these were developed more than three decades ago, the Rutter questionnaires do not adequately cover many areas of contemporary interest such as concentration, impulsivity, victimisation, and prosocial behaviour. Furthermore, there is no version of the Rutter questionnaire that can be completed by children and adolescents themselves. The Achenbach questionnaires are better in this respect as they include versions for completion by parents - the Child Behaviour Checklist (CBCL), teachers - the Teacher Report Form (TRF), and youth themselves - the Youth Self-Report (YSR). CBCL, TRF, and YSR intend to cover a broad range of behavioural and emotional symptoms that are particularly relevant when assessing children and adolescents in clinical settings [1]. Yet, for screening or for research purposes, the Achenbach questionnaires are less useful as they are long and contain many items that are not relevant to the majority of children [1].

However, a new behavioural screening instrument, the Strengths and Difficulties Questionnaire (SDQ), was recently developed from the Rutter scales by the English child psychiatrist Robert Goodman. In line with ideas of contemporary child psychiatry and child psychology, the SDQ covers areas insufficiently accounted for by the older Rutter forms, eg. attention and peer relations [2, 3].

Therefore, SDQ was selected as an instrument to measure mental health problems of Tamil-speaking adolescents in Sri Lanka. There are two versions of SDQ: informant rated version where parents or teachers complete the questionnaire and the self-report version where the adolescents themselves complete the questionnaire $[4,5]$. The SDQ self-report was translated and validated in this study.

\section{Methods}

The SDQ presents 25 attributes, some positive and others negative; respondents use a 3-point Likert scale to indicate how far each attribute applies to them. The 25 items are sorted in five groups of five items each, generating scores for emotional symptoms, conduct problems, hyperactivity-inattention, peer problems, and pro-social behaviour; all but the last are summed to generate a total difficulties score [4]. Each item can be marked 'not true', 'somewhat true', or 'certainly true' and is transferred into a score of 0,1 or 2 . The score for each of the five scales is generated by summing the scores for the five items that make up the scale, thereby generating a scale score ranging $0-10$. The scale scores for hyperactivity, emotional symptoms, conduct problems and peer problems can be added to a total difficulties score ranging $0-40$. The pro-social score is not incorporated in the reverse direction into the total difficulties score since the absence of pro-social behaviours is conceptually different from the presence of psychological difficulties.

The SDQ self-report was translated from English to Tamil and back translated to English. Translation and back translation were carried out by competent people who are fluent in both languages. Consensus was reached for differences between original and back translation. Final translation is available on the web: http:// www.sdqinfo.com.

Content validity (each item is to assess a content that is relevant to each culture under investigation), semantic validity (words used in the original and translation must have a similar meaning), and conceptual validity (responses to an interview relate to a theoretical construct within the culture) of the instrument were achieved during the translation process described above [6]. There are no other diagnostic instruments available in Tamil against which SDQ can be validated on the aspect of convergent validity. Criterion validity of the translated SDQ was tested among young adolescents attending a psychiatric clinic and in a community sample. Diagnosis made by the consultant psychiatrist was considered the gold standard to test the criterion validity.

Thirty-five patients aged 11-16 years attending the psychiatric clinic of the Teaching Hospital, Jaffna for the first time were included for validation purposes. Patients presenting with acute psychosis and mentally retarded adolescents were excluded. Adolescents and their parents or guardians were informed about this study and verbal consent was obtained. Adolescents were asked to complete the SDQ self-report. After completing the SDQ they were examined by the consultant psychiatrist to arrive at a diagnosis.

To test the criterion validity in the community sample it was not possible for the consultant psychiatrist to go to the field due to his heavy workload. Consequently, 91 school going 11 -16 year old adolescents were recruited as a convenience sample through the area Family Health Worker. After obtaining informed consent, they were asked to complete the SDQ followed by a semi-structured interview by a psychologist who has 5 years of experience in the child mental health clinic in the Teaching Hospital, Jaffna. The interview notes were later discussed with the consultant psychiatrist to decide the presence or absence of mental health problems.

A representative sample of 1199 adolescent school children of 14-15 years of age were given the SDQ self report. Reliability of SDQ was estimated using internal consistency.

Validity of the SDQ was derived using receiver operating characteristic (ROC) curves and identifying sensitivity and specificity for various cut-off points. The ability of the SDQ scores to distinguish between 'normal mental health' and 'mental health problems' (as diagnosed 
by consultant psychiatrist) was examined using ROC curves, employing the area under the curve (AUC) as the index of ability to discriminate [7]. As a guide to interpretation, the area under a ROC curve is 1.0 for a measure that discriminates perfectly, and 0.5 for a measure that discriminates with no better than chance accuracy [3]. It was assumed that increasing numbers on the SDQ scale represent the increasing belief that the subject has mental health problems, while decreasing numbers on the SDQ scale represent the increasing belief that the subject has normal mental health.

Reliability of SDQ was measured using internal consistency, which measures the consistency of results across items within a single test $[2,8,9]$. Generally this method is used where participants respond to questions on a scale. SDQ has five scales (emotional, conduct, hyperactivity, peer problem and pro-social), and each scale is composed of five items. Internal consistency measures how well each item in a sub-scale relates independently to the rest of the items on the scale and how they are related overall. A scale with an alpha above 0.6 is considered as internally consistent. Cross-scale correlation was performed to assess the correlation between each of the symptom scales.

\section{Results}

Thirty-five adolescents from the psychiatric clinic were diagnosed as having mental health problems. Among the 95 students in the community sample, 27 were found to have mental health problems and 64 were identified as normal. Altogether, there were 62 adolescents with mental health problems and 64 adolescents with normal mental health. The ROC curve was drawn with the SDQ total difficulties score as the test variable and the diagnosis made by the consultant psychiatrist as the state variable (figure1). The area under the curve is 0.87 (95\% - CI = $0.79-0.93)$. The co-ordinates for various cut-off points in total difficulties score of SDQ are given in table 1 . International cut-off point of 15 gave a sensitivity of $69.4 \%$ and specificity of $92.2 \%$.

Internal consistency coefficients (Cronbach's alphas) for various SDQ scales were generally satisfactory (0.67-0.78) (table 2). Cross-scale correlation showed that individual symptom sores of the difficulties scale (emotional, hyperactivity, conduct, and peer problem) have good positive correlation with the total score as well as among each other. Pro-social score has a negative correlation with the total difficulties score and individual difficulties symptom scores (table 3 ).

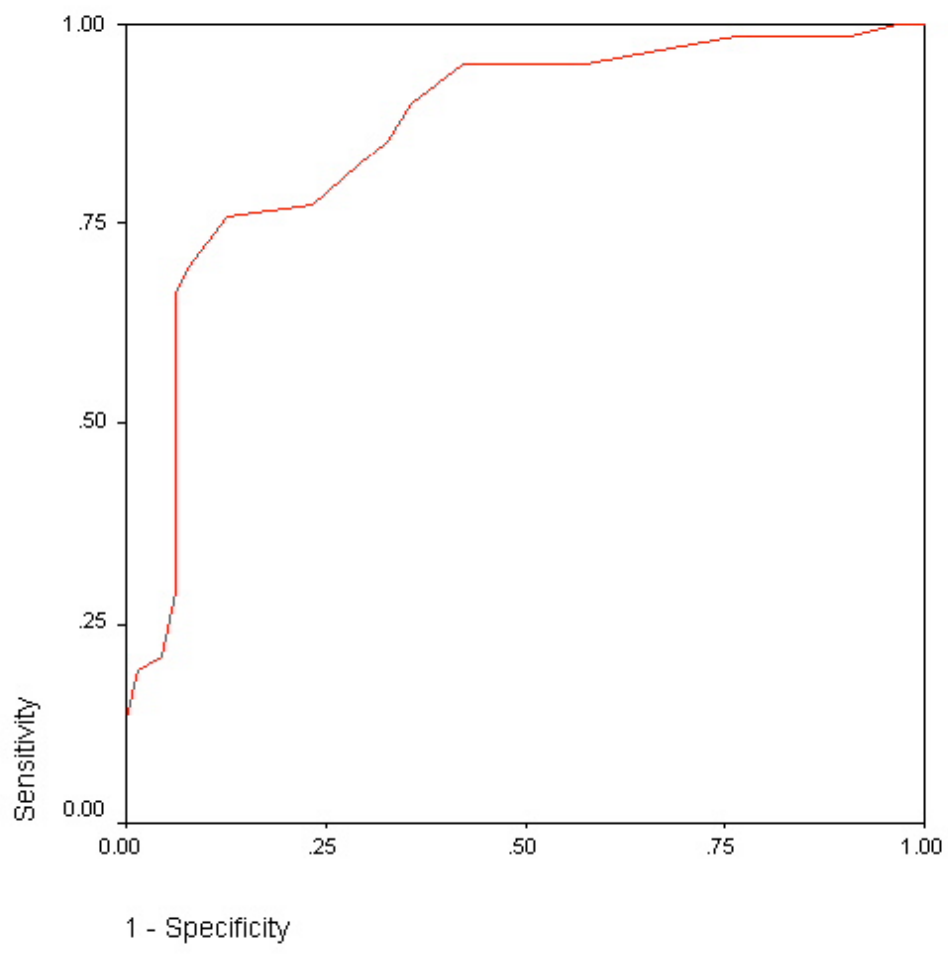

Figure 1. ROC curve showing sensitivity against 1 -specificity (n: 126) 
Table 1. Predictive values of SDQ score for various cut-off points $(n=126)$

\begin{tabular}{ccccccc}
\hline Cut-off & Sensitivity & Specificity & $\begin{array}{c}\text { Positive } \\
\text { predictive } \\
\text { value }\end{array}$ & $\begin{array}{c}\text { Negative } \\
\text { predictive } \\
\text { value }\end{array}$ & Normal & Abnormal \\
\hline 12 & 85.5 & 67.2 & 71.6 & 82.7 & 52 & 74 \\
13 & 77.4 & 76.6 & 76.2 & 77.8 & 63 & 63 \\
14 & 75.8 & 87.5 & 85.5 & 78.9 & 71 & 55 \\
15 & 69.4 & 92.2 & 89.6 & 75.6 & 78 & 48 \\
16 & 66.1 & 93.7 & 91.1 & 74.1 & 81 & 45 \\
17 & 51.6 & 93.7 & 88.9 & 66.7 & 90 & 36 \\
\hline
\end{tabular}

Table 2. Cronbach's alpha coefficients of the SDQ scores $(n=1199)$

\begin{tabular}{lc}
\hline Scale & Alpha \\
\hline Total score & 0.78 \\
Emotional & 0.73 \\
Conduct & 0.70 \\
Hyperactivity & 0.72 \\
Peer problem & 0.67 \\
Pro-social & 0.73 \\
\hline
\end{tabular}

Table 3. Cross-scale correlations of SDQ scores $(n=1199)$

\begin{tabular}{lcccccc}
\hline Scale & Total & Emotional & Conduct & Hyperactivity & Peer & Pro-social \\
\hline Total score & 1.0 & & & & & \\
Emotional & 0.73 & 1.0 & & & & \\
Conduct & 0.69 & 0.32 & 1.0 & & & \\
Hyperactivity & 0.75 & 0.36 & 0.44 & 1.0 & & \\
Peer problem & 0.63 & 0.27 & 0.24 & 0.33 & 1.0 & \\
Pro-social & -0.32 & -0.04 & -0.29 & -0.34 & -0.24 & 1.0 \\
\hline
\end{tabular}

\section{Discussion}

There is no validated instrument to measure mental health of Tamil-speaking adolescents in Sri Lanka. A majority of them live in conflict affected areas where mental health services are scarce and mental health problems are more $[10,11]$. Identifying a valid tool to screen mental health problems of adolescents living in these areas is important. SDQ was selected among other instruments to measure mental health of adolescents, because SDQ is only a one-page questionnaire with 25 questions and easy to use [5]. Moreover, SDQ is also available free of charge for non-commercial purposes [1].

In developed countries such as the UK, SDQ was validated against another psychiatric instrument or a structured detailed clinical interview $[3,4,12]$. Absence of another validated instrument in Tamil to compare the SDQ, limits the use of convergent validity in the local setting. Therefore, SDQ was tested using criterion validity. The diagnosismade by the consultant psychiatrist was considered the gold standard. Ideally, the study could have been carried out using at least two adolescent psychiatrists to assess the adolescents. There was no other consultant psychiatrist available in the local setting to verify the diagnosis considered as the gold standard. Another limitation was the use of two different methods in assessing community and clinic samples. It was not possible to take the only psychiatrist available in the 
district to the field compromising his clinical duties or bring apparently normal children to the mental health clinic due to the stigma associated with mental illnesses. In addition to criterion validity, cultural appropriateness of the instrument was achieved during the translation process. Despite these limitations, the results are promising for the validity and reliability of SDQ self-report as a screening instrument in Tamil.

\section{Acknowledgement}

This research is funded by the Sida/SAREC grant for Health and Social Care for Socially Marginalised People in Sri Lanka.

\section{References}

1. Muris P, Meesters C, Eijkelenboom A, Vincken M. The self-report version of the strengths and difficulties questionnaire: its psychometric properties in 8- to 13-yearold non-clinical children. British Journal of Clinical Psychology 2004; 43: 437-48.

2. Muris P, Meesters C, van den Berg F. The strengths and difficulties questionnaire (SDQ) - further evidence for its reliability and validity in a community sample of Dutch children and adolescents. European Child and Adolescent Psychiatry 2003; 12: 1-8.

3. Malmberg M, Rydell AM, Smedje H. Validity of the Swedish version of the strengths and difficulties questionnaire (SDQ-Swe). Nordic Journal of Psychiatry 2003; 57: 357-63.

4. Goodman R. Psychometric properties of the strength and difficulties questionnaire. American Academy of Child and Adolescent Psychiatry 2001; 40.

5. Goodman R. The strengths and difficulties questionnaire: A research note. Journal of Child Psychology and Psychiatry 1997; 38: 581-6.
6. Sumathipala A, Murray J. New approach to translating instruments for cross cultural research: a combined qualitative and quantitative approach for translation and consensus generation. International Journal of Methods in Psychiatric Research 2000; 9: 88-95.

7. Van Widenfelt BM, Goodman R, Goedhart AW, Treffers PDA. Dutch version of strengths and difficulties questionnaire (SDQ). European Child and Adolescent Psychiatry 2003; 12: 281-9.

8. Becker A, Woerner W, Hasselhorn M, Banaschewski T, Rothenberger M. Validation of the parent and teacher SDQ in a clinic sample. European Child and Adolescent Psychiatry 2004; 13: 11-6.

9. Karen H, Bournon RG, Donald S, Simpson G. The strengths and difficulties questionnaire: U.S. normative data and psychometric properties. Journal of American Academy of Child and Adolescent Psychiatry 2005; 44: 557-64.

10. Somasundaram DJ. Child soldiers: understanding the context. British Medical Journal 2002; 324: 1268-71.

11. Somasundaram DJ. Psychosocial Consequences of War. New York: Kluwer Academic 2002.

12. Marzocchi GM, Capron C, Di Pietro M, Tauleria E, Duyme M, Frigerio A, Gaspar MF, Hamilton H, Pithon G, Simoes A, Therond C. The use of the strengths and difficulties questionnaire (SDQ) in southern European countries. European Child and Adolescent Psychiatry 2004; 13 (Suppl 2): 1140-6.

13. Goodman R, Renfrew D, Mullick M. Predicting the type of disorder from strengths and difficulties questionnaire scores in child mental health clinics in London and Dhaka. European Child and Adolescent Psychiatry 2000; 9: 129-34.

14. Goodman R, Ford T, Simmons H, Gatward R, Meltzer H. Using the strengths and difficulties questionnaire (SDQ) to screen for child psychiatric disorders in a community sample. International Review of Psychiatry 2003; 15: 166-72.

15. Rutter M. Psychosocial influences: critiques, findings, and research needs. Development and Psychopathology 2000: 375-405. 